\title{
Regulating the Scope of EMS Services
}

\author{
Paul E. Pepe, MD, FACP, FACEP, FCCP, $*$ Marni J. Bonnin, MD, ${ }^{+}$ \\ Kenneth L. Mattox, MD, FACS ${ }^{++}$
}

\section{Introduction}

Esse quam videri

The issue of what is the proper "scope of practice" for today's paramedics continues to surface..$^{1-3}$ Recently, this has prompted discussions by regulatory and legislative bodies. ${ }^{2}$ It seems that the issue arises out of a concern that many EMS systems in the United States may not be well-controlled medically and that paramedics may be performing many procedures which have not been validated scientifically for prehospital emergency medical care. It is asserted that some of the procedures practiced by paramedic services may be unnecessary or even deleterious. Furthermore, some believe that the performance of some procedures not only is unnecessary but also delay the provision of definitive care in the hospital. ${ }^{1-3}$ Others hold that paramedics do not have sufficient clinical experience to allow recognition of subtle historical details and important, atypical, clinical symptoms and signs. Therefore, they have the potential to administer powerful drugs inappropriately or fail to detect complications associated with their administration. Hence, there are contentions that the "scope of practice" of paramedics should be limited through changes in the laws and the regulations which govern such practices. However, we contend that limiting the paramedic's scope of services through legislative prohibition of prehospital procedures is a short-sighted approach to the issues associated with alledged inappropriate prehospital emergency medical care. A more appropriate solution would be to strengthen and intensify proper medical supervision of EMS systems.

The provision of EMS, despite all of the modern-day trappings and political considerations, is the practice of medicine in the prehospital setting. ${ }^{+10}$ Although the day-to-day care and procedures are delivered directly by intermediaries (e.g., paramedics, EMTs), essentially all legislative bodies in the United States recognize that such acts are delegated to these surrogates by designated, accountable physicians. Therefore, when one talks about limiting the "paramedic's scope of practice," it translates into limiting the accountable physician's scope of practice. Perhaps a more appropriate solution would be to scrutinize the practice of the EMS physicians. When medical supervision is provided properly, paramedic "practice" no longer is an issue.

\section{The Roots of EMS}

When the prototypes for today's EMS systems first were launched only two decades ago, generally they were research projects carried out by small groups of academic physicians in a few centers. These unique doctors, responding into the community, rapidly became adept at "hands-on" emergency care in the out-ofhospital setting where the logistics and environment modified their patient care

\footnotetext{
*Associate Professor of Medicine and Surgery, Baylor College of Medicine, Director of EMS-City of

Houston, Texas, USA, President-National Association of EMS Physicians

+Assistant Professor of Medicine, Baylor College of Medicine, Assistant Director of EMS-City of

Houston, Texas, USA

++Professor of Surgery and Director of Trauma Services, Baylor College of Medicine, Harris County

Hospital District, Houston, Texas, USA
} 
strategies, priorities, and judgments. ${ }^{4,5}$ Subsequently, recognizing that many of their prehospital interventions not only were efficacious, but that many of these timecritical, life-saving procedures could be performed by accompanying rescue personnel, programs soon were developed specifically to train them as specialized "emergency medical technicians" (EMTs). ${ }^{7.8}$ Therefore, the original physician supervisors not only were privy to the unique aspects of prehospital care, they also directly passed on the "tricks of the trade" to their new apprentices. ${ }^{4.8}$ Furthermore, the care provided by these new "EMS" personnel clearly was considered an extension of that supervising physician's practice.

The caveats for such activities were nested in the facts that: 1) essentially, the original EMS physicians had "lived" with their surrogates day and night in the streets, in some case for as much as a year; 2 ) as a result, they knew and trusted them with the delivery of specific modalities of assessment and care; and 3) they were so comfortable with the abilities of these new patient care providers that they would bet their medical licensure on them. The involvement of the early pioneers in these systems for which they accepted total accountability for all medical actions was profound. ${ }^{10}$

These programs were successful and were able to demonstrate life-saving effects. And, ongoing success was ensured by these physicians who continued to scrutinize the performance of their surrogates on a continuous and ongoing manner, with nearly 24-hour radio monitoring, frequent on-scene observation, and rigorous scientific analysis of patient care procedures whenever possible. ${ }^{4.6 .8 .10}$ Regardless of the size and scope of the systems for which they were responsible, the commitment and involvement required to effect such programs were and remain enormous. Such physicians hold a public trust that each patient seen by EMS personnel is receiving the same level of care that he or she would have delivered had they been physically present on the scene. Unfortunately, many EMS systems, even those now provided with full-time medical directors, have evolved without such strong foundations. Often, this has created obstacles to EMS system improvements in that the unlearning of bad clinical habits created in a void may be a more difficult task to accomplish than starting out fresh.

\section{Fine Wine Doesn't Always Travel Well}

The early EMS successes soon were emulated by many other communities. However, the results for some of these programs seem less impressive to me than were those of their predecessors. While EMT and paramedic training curricula were codified like recipes, and ambulances and other equipments were purchased at great public expense, many systems seem to have fallen far short of their potential. Often, the principal missing ingredient was (and is) the intense involvement and supervision of a committed medical director. Unfortunately, rather than prehospital EMS being the practice of publicly accountable, street-wise medical directors, often the medical practice essentially is controlled by committees of "interested" physicians (and others) who issue recipes for care yet do not know the actual capabilities, strengths, and weaknesses of the practitioners. Furthermore, it is rare that any of these delegators of care ever have practiced in the often hostile prehospital environment. In other situations, medical support is provided by physician "advisors" who not only never have practiced in this setting, but who never have witnessed the capabilities of the paramedics let alone trust them to do exactly as they would in a similar, critical situation. Quite bluntly, the primary critics of prehospital emergency care personnel always seem to be persons who cannot identify with the problems which are peculiar to the provision of prehospital emergency medical care.

Recently, many states have attempted to deal with this problem by legislating that EMS systems must be supervised closely by medical directors who are experienced in the delivery of prehospital EMS. Unfortunately, often such appointments are politically or 
financially determined rather than based on ability and experience. In other cases, these appointees are well-intentioned, volunteer physicians who are not compensated for their endeavors and who even may have neglected career opportunities and their families in favor of their "public" responsibilities. This is complicated further by the fact that few have had role models upon which to base their activities. Thus, talented and dedicated physicians may remain relatively ineffective and unable to appreciate all of the complexities and commitments required to provide the medical leadership for the programs in which they operate. Eventually, such people either relinquish their EMS activities or just maintain the status quo and fail to provide bold new direction. They become passive medical directors and the EMS system becomes a costly medical transport system.

\section{The Essential Element in EMS}

As with any other medical disciplines, EMS deserves close medical scrutiny to confirm efficacy. When the general performance and effectiveness of various EMS systems in the United States are evaluated, there is an apparent positive correlation with the amount of direct involvement of physicians. There are sophisticated systems which have been operational for more than a decade in which negligible successful resuscitation rates from cardiac arrest increased dramatically following the introduction of a single factor-a "streetwise" and committed medical director. ${ }^{11}$ A good prehospital EMS system operates with good radios, good vehicles, good medical directors, good defibrillators, and good paramedics and EMTs. In other words, medical directors should be standard and essential components of an EMS system and their costs must be a line item in the EMS budget.

Beyond this, EMS is a very busy practice. Most EMS systems can expect one true emergency call per 10,000 population every 24 hours. ${ }^{8}$ In urban areas, EMS organizations usually respond to 50 to 60 injuries and three or four cardiac arrests per million population daily. ${ }^{9}$ It is clear that the full-time involvement of several physicians becomes a requirement in large urban centers if a high standard of practice is expected at each scene.

Today, progressive EMS systems make full-time physician involvement a priority. Municipalities, particularly, have begun to hire full-time physician directors for their EMS systems. ${ }^{5}$ Generally, municipalities and medical schools in large urban centers have led the way in conjointly sponsoring such endeavors. In some major cities, several physicians are funded for full-time EMS activities. In small municipalities, fulltime commitments may be unnecessary, but partial compensations for time dedicated to EMS should be part of the EMS budget. In addition, many academic centers provide residents and fellows with dedicated EMS experience to better ensure prehospital care training and research as well to facilitate better role-modeling for EMS supervision. ${ }^{10,12}$

\section{Who's to Blame?}

The initial reaction when something has gone wrong is to find who's at faultnot why he or she is at fault. When patients repeatedly arrive at the emergency department with an endotracheal tube placed in the esophagus, it is easy to lay blame elsewhere and become nervous about liability (especially if you're the physician who ordered that the tube be placed). The initial reaction would be to remove privileges from the paramedic(s) or to openly damn the whole system as incompetent. We believe that if the involved physicians originally had trained the paramedics appropriately, constructively modified their field behavior, and carefully monitored the performance of the system, it is likely that the errors would not have ever occurred..$^{13}$

Similarly, for a physician to state globally that IVs are unwarranted in prehospital "trauma management" because they delay transport is an over-generalization, can be 
somewhat misleading, and actually wrong in certain cases. Likewise to withhold certain drugs from patients who clearly need them (e.g., furosemide in fulminant congestive heart failure) because a base-station doctor is uncertain about a paramedic's ability to evaluate and manage the case also is unfortunate. If the paramedic was incapable, whose fault was it-the trainEE or the trainER?

Furthermore, physician groups have a propensity to generate guidelines and consensus formulas to dictate proper therapeutic priorities. This has been true particularly for the prehospital emergency setting. However, like any other discipline in medicine, medical practice requires individual attention for each patient. It is not possible to fit the thousands of scenarios into nice pigeon holes defined by such protocols. Clearly, just as in the in- hospital setting, guidelines are just guidelines. They do not substitute, for intensive training and years of clinical experience. Many successful systems, as indicated by successful resuscitations and other quality assurance measures, use little in the way of protocols but instead depend upon nearly round-the-clock, intense physician oversight and this means that the physician providing on-line medical direction actually has been "out there" many times, knows the idiosyncrasies of the individual paramedics, understands the particular logistics associated with the situation, and therefore recognizes the unique features of the particular case. Also, what is done in one EMS system may not be appropriate in another. Transport times, logistical ability to supervise, and other factors change the needs and the level of comfort with which an accountable physician can live. In one setting, a medical director may be able to train paramedics to perform successful tracheotomies, while in another setting, the same director may be unable to feel comfortable with his or her trainees.

While many EMS programs try to dictate "better" medical care through the use of protocols, the effect may be paradoxical. Few trauma protocols delineatethe treatment for a gunshot wound to the lower leg from one to the abdomen or the differences in treating an altered conscious state following a long fall from that secondary to a gunshot wound to the head. Clearly, priorities will differ. Moreover, protocols usually are too generic and a paramedic is accosted by one physician for not performing a procedure only to be reprimanded by another for performing the procedure in another similar case. This leads to frustration and confusion when such caveats for care are not explained and clarified by supervising physicians. This "damned if you do, damned if you don't" situation leaves paramedics resentful, recalcitrant, and predisposed to "go around" the system.

The concept of "loss of medical control" really is a loss of the credibility of the physicians responsible for the same. When medical direction is provided by knowledgeable physicians who understand the subtle differences in strategies for various prehospital cases and the limitations of the settings in which the care is being delivered, and who consistently give appropriate feedback to the paramedics, "medical control" is a moot issue. 5.6 Although such persons are hard to find, the provision of medical direction by these individuals is worth it. Most paramedics are dedicated, hard-working individuals who, with the right leadership and direction, can be inspired to perform almost any difficult medical task and are willing to accept constructive criticism which could result in improved patient care. In fact, what often comes from such involvement of the medical director and the paramedics is scientific scrutiny of the care, enhanced morale, and diminished complications. And the intangibles of improved paramedic morale, behavior, and compassion, learned by example, simply lead to better patient care. It is the level of involvement that is the key determinant for each of the above. The payback - an excellent EMS system-is worth it. 


\section{The (Real) Future of EMS}

In light of the above, one more caveat must be emphasized-the training of the prehospital emergency medical services physicians. It is possible that one can pay a physician to work EMS full time and give him or her a whole set of written roles and responsibilities such as "review charts" and "on-scene supervisions." But without good role models for medical directorship, even eager, well-intentioned physicians may not be as effective as they should. ${ }^{+}$The future of EMS systems and the delivery of prehospital emergency medical care will depend on our ability to provide excellent physician role models as well as to formally and properly train the EMS physicians of tomorrow..$^{4.12}$ And, as long as meticulous attention to detail, boldness, and compassion are part of the formula, the future of EMS holds great promise. Instead of arguing about limiting the scope of paramedic practice, we will be researching ways in which to broaden it.

\section{References}

1. Smith JP, Bodai BI: The urban paramedic's scope of practice. JAMA 1985; 253:544-548.

2. McCallion R: Paramedic scope of practice under fire. The Protocal. San Francisco Paramedic Association, San Francisco, Calif, June/July 1987.

3. Smith JP, Bodai BI, Hill AS: Prehospital stabilization of critically injured patients: A failed concept. Presentation at the 43rd annual meeting of the American Association for the Surgery of Trauma, October 1983.

4. Pepe PE; Past, present, and future of emergency medical services. Prehospital and Disaster Medicine $1989 ; 4: 47-49$.

5. Pepe PE, Stewart RD: Role of the physician in the prehospital setting. Ann Emerg Med 1986; 15:1480-1483.

6. Stewart, RD: Medical direction in emergency medical services: the role of the physician. Emerg Med Clin North Am 1987; 5:119-132.

7. Page JO: The Paramedics. Morristown, New Jersey: Backdraft Publications, 1979.

8. Cobb LA, Alvarez H, Copass MK: A rapid response system for out-of-hospital cardiac emergencies. Med Clin North Am 1976; 60:283-290.

9. Pepe PE, Copass MK. Prehospital care. In: Early Care of the injured. American College of Surgeons Committee on Trauma. EE Moore (ed), Philadelphia; B.C. Decker, Inc, 1990.

10. Copass MK: The urban experience in medical control. In: Proceedings of the Subcommittee on Medical Control, Committee on Emergency Medical Services, Assembly of Life Sciences National Research Council, National Academy of Sciences. Washington, DC: National Academy Press, 1981; 92-94.

11. Pepe PE, Bolenbaucher AW, Mattox KL, et al: Impact of intense physician supervision on the effec tiveness of an emergency medical services system. Ann Emerg Med 1988; 17:752.

12. Paris PM, Stewart RD: Survey of requirements for EMS experience in emergency medicine residents. Ann Emerg Med 1986; 15:619.

13. Pepe PE, Copass MK, Joyce TH: Prehospital endotracheal intubation rationale for training emergency medical personnel. Ann Emerg Med 1985; 14:1085-1092. 